\title{
The Detection of Bovine Lactoferrin Binding Protein on Trypanosoma brucei
}

\author{
Tetsuya TANAKA ${ }^{1)}$, Yukie ABE ${ }^{1)}$, Noboru INOUE ${ }^{2)}$, Woan-Sub KIM ${ }^{1)}$, Haruto KUMURA ${ }^{1)}$, Hideyuki NAGASAWA ${ }^{2)}$, \\ Ikuo IGARASHI $^{2)}$ and Kei-ichi SHIMAZAKI ${ }^{1)}$ \\ ${ }^{1)}$ Dairy Science Laboratory, Graduate School of Agriculture, Hokkaido University, Sapporo, Hokkaido 060-8589 and ${ }^{2)}$ The Research \\ Center for Protozoan Diseases, Obihiro University of Agriculture and Veterinary Medicine, Obihiro, Hokkaido 080-8555, Japan
}

(Received 7 July 2003/Accepted 16 January 2004)

ABSTRACT. Trypanosoma brucei, the causative agent of sleeping sickness in humans, requires transferrin (TF) for growth. Therefore, $T$. brucei has a TF receptor that allows it to obtain iron from TF. Lactoferrin (LF), a member of the TF family protein, is an iron-binding protein that is found in most biological fluids of mammals. LF has been shown to interact with some bacteria species by specifi $\mathrm{c}$ receptor-ligand binding. We examined the ability of T. brucei to bind bovine LF (bLF) by using a fluorescence test, streptavidin-biotin (SAB) microplate analysis, and far Western blotting using a biotin-streptavidin system. We found that bLF bound to components of T. brucei, and that bLF hydrolysate disrupted the sites responsible for binding to parasite proteins. Furthermore, bLF, human LF, bovine TF, and ovotransferrin bound same proteins of $T$. brucei, which exhibited molecular masses of 40 and $43 \mathrm{kDa}$. The N-terminal amino acid sequence of the $40 \mathrm{kDa}$ bLF binding protein was identified as glyceraldehyde-3-phosphate dehydrogenase (GAPDH).

KEY WORDS: glyceraldehyde-3-phosphate dehydrogenase, lactoferrin, ovotransferrin, transferrin, Trypanosoma brucei.

J. Vet. Med. Sci. 66(6): 619-625, 2004

The flagellated protozoan parasite, Trypanosoma brucei, which is transmitted by the bite of the tsetse fly, is the causative agent of sleeping sickness in humans and Nagana in livestock. Within the mammalian host, trypanosomes live and multiply extracellularly in the blood and tissue fluids. Like all other living organisms, bloodstream forms of $T$. brucei require iron for growth, although this life-cycle stage lacks cytochromes. All iron that bloodstream trypanosomes need for propagation is delivered by the host's transferrin (TF) $[19,24]$. The parasite internalizes host TF by receptormediated endocytosis. This process is 200 times faster than the rate of pinocytosis [3]. The trypanosomal receptor for TF uptake is an unusual protein, which bears no structural similarity to its mammalian counterpart.

Lactoferrin (LF), a cationic iron-binding protein that is produced and secreted by mammary glands and neutrophils, exhibits a broad spectrum of antimicrobial properties [8, 22] including the activation of natural killer cytotoxicity against tumor cells $[7,16]$. LF has been shown to interact with some microorganisms by specific receptor-ligand binding $[2,20,21]$. Because protozoan parasites require iron for growth and development, the sequestration of free iron by LF may be one of important nonspecific host defense mechanisms that starve microorganisms of this essential element. Therefore, some microorganisms, including trichomonads, have adapted to using LF as an iron source. The vaginal tract pathogens Trichomonas vaginalis and $T$. foetus acquire iron from LF via a specific 136 or $155 \mathrm{kDa}$ receptor, respectively, found on the cell surface [4, 12]. Furthermore, a 70 $\mathrm{kDa}$ TF binding protein was previously identified on the surface of several Leishmania spp. including L. infantum, which is thought to be closely related to L. chagasi [28]. However, previous studies have focused mainly on TF interaction with $T$. brucei, and little information is available on the interaction of LF with T. brucei. Based on previous studies on other protozoan parasites, we expected that LF would also function as a bridge molecule between $T$. brucei and iron. Therefore, in the present study, we examined the specific $T$. brucei protein to which LF binds and compared it with those that bind TF and ovotransferrin (OTF).

\section{MATERIALS AND METHODS}

Chemicals: Complete protease inhibitor (Boehringer Mannheim, Mannheim, Germany), N-hydroxysuccinimide biotin, $O$-phenylenediamine (Sigma Chemical Co., St. Louis, MO), fluorescein conjugated-avidin (avidin-FITC), horseradish peroxidase conjugated-streptavidin (streptavidin-HRP) (Nichirei Co., Tokyo, Sigma Chemical Co.), bovine serum albumin (BSA), bovine TF, and OTF (ironfree type, Sigma Chemical Co.) were used. Bovine irondeficient (apo)-LF (bLF) and iron-saturated (holo)-bLF (Morinaga Milk Co., Tokyo) were prepared according to Law and Reiter [11]. Bovine LF hydrolysate by pepsin was supplied from Morinaga Milk Co. The ECL reagent kit (Amersham Bioscience Co., Piscataway, NJ) was used to detect chemiluminescence.

Parasites: Bloodstream forms (BDFs) of $T$. $b$. rhodesiense IL2343, T. b. gambiense, and T. evansi, were obtained from International Livestock Research Institute, Nairobi, Kenya. The parasite culture was centrifuged and the supernatant was removed. The pelleted parasites were counted using a hemocytometer and washed three times with phosphate-buffered saline (PBS). Finally, parasites were distributed into fresh tubes at a ratio of $1 \times 10^{9}$ per tube. The tubes containing the parasites were stored at $-80^{\circ} \mathrm{C}$ until use in the following experiments.

Parasite lysis: Soluble proteins were extracted from parasites using the following method. The cell pellets were suspended in $50 \mu l /$ tube of TNE buffer $(50 \mathrm{mM}$ Tris- $\mathrm{HCl} \mathrm{pH}$ 
7.5, $100 \mathrm{mM} \mathrm{NaCl}, 2 \mathrm{mM}$ EDTA pH 8.0), RIPA buffer (10 $\mathrm{mM}$ Tris- $\mathrm{HCl} \mathrm{pH}$ 7.4, $150 \mathrm{mM} \mathrm{NaCl}, 5$ mM EDTA pH 8.0, $1 \%$ TritonX-100, $0.1 \%$ SDS) containing $2 \mu \mathrm{l}$ complete protease inhibitor or SDS lysis buffer (50 mM Tris- $\mathrm{HCl} \mathrm{pH} \mathrm{7.5,}$ $0.5 \%$ SDS, $1 \mathrm{mM}$ DTT). After an overnight incubation at $4^{\circ} \mathrm{C}$, the cell lysates were centrifuged at $11,000 \mathrm{~g}$ for $30 \mathrm{~min}$ to obtain supernatants containing the soluble fraction.

Biotinylation of proteins: To prepare biotinylated proteins, N-hydroxysuccinimide biotin was dissolved at a concentration of $10 \mathrm{mg} / \mathrm{ml}$ in dimethylsulfoxide and the proteins (bLF, bTF, and OTF) were dissolved at a concentration of $3 \mathrm{mg} / \mathrm{ml}$ in $0.1 \mathrm{M}$ sodium borate buffer ( $\mathrm{pH} \mathrm{8.8)}$ ). The biotin ester was then added at a ratio of $250 \mu \mathrm{g}$ of biotin ester per $1 \mathrm{mg}$ of protein, mixed well and incubated at room temperature for $4 \mathrm{hr}$. Finally, $20 \mu \mathrm{l}$ of $1 \mathrm{M} \mathrm{NH}_{4} \mathrm{Cl}$ per 250 $\mu \mathrm{g}$ of biotin ester was added, and the solution was incubated at room temperature for $10 \mathrm{~min}$. After incubation, the reaction mixture was dialyzed extensively against PBS to remove the unreacted reagent. The estimated biotinylation ratio was $32 \%$ of total amino groups based on the molecular mass measured by matrix assisted laser desorption ionization-time of flight mass spectroscopy.

Binding on T. brucei assayed by fluorescence test: Trypanosoma brucei BDFs were fixed with methanol and incubated with biotinylated bLF (bLF-bio), biotinylated bTF (bTF-bio), or biotinylated OTF (OTF-bio) at $37^{\circ} \mathrm{C}$ for 30 min at a concentration of $10 \mu \mathrm{g} / \mathrm{ml}$ in dilution buffer containing $3 \%$ BSA and $0.05 \% \mathrm{NaN}_{3}$ in PBS. The cells were then washed three times with PBS and incubated in avidinFITC at $37^{\circ} \mathrm{C}$ for $30 \mathrm{~min}$, which was diluted 200 times with the dilution buffer. The cells were again washed three times and observed under a TCS-NT confocal laser scanning microscope (Leica Geosytems, Tokyo).

Binding on T. brucei assayed by streptavidin-biotin $(S A B)$ microplate method: Ninety-six well microtiter plates (Corning Inc., Corning, NY) were coated with $50 \mu l$ of $T$. bruce $i$ BSF lysate $\left(1 \times 10^{8} / \mathrm{m} l\right)$ in $0.05 \mathrm{M}$ carbonate-bicarbonate buffer ( $\mathrm{pH} 9.6)$ overnight at $4^{\circ} \mathrm{C}$, washed once with PBS containing $0.05 \%$ Tween 20 (PBST), and blocked with $100 \mu \mathrm{l}$ of PBS diluted in $1 \% \mathrm{BSA}$ for $1 \mathrm{hr}$ at $37^{\circ} \mathrm{C}$. After washing with PBST, bLF-bio, bTF-bio, or OTF-bio were added at a concentration of $10 \mu \mathrm{g} / \mathrm{m} l$ to each well in $50 \mu l$ volumes. The plates were incubated for $1 \mathrm{hr}$ at $37^{\circ} \mathrm{C}$ and washed six times with PBST. A $50 \mu l$ aliquot of streptavidin-HRP diluted at 1:10,000 was added to each well and again incubated for $1 \mathrm{hr}$ at $37^{\circ} \mathrm{C}$. The wells were washed with PBST six times, and $100 \mu l$ of the substrate solution (0.1 $\mathrm{M}$ citric acid, $0.2 \mathrm{M}$ sodium phosphate, $0.003 \% \mathrm{H}_{2} \mathrm{O}_{2}$ and $0.4 \mathrm{mg} / \mathrm{ml} O$-phenylenediamine) were used for color development. After $1 \mathrm{hr}$, the optical density (O. D.) was read at $492 \mathrm{~nm}$ on a MTP-120 plate reader $(\mathrm{TOSOH}$, Tokyo).

Competitive inhibition assayed by $S A B$ microplate method: Ninety-six well microtiter plates (Corning Inc., Corning, NY) were coated with $50 \mu l$ of $T$. brucei BSFs lysates $\left(1 \times 10^{8} / \mathrm{ml}\right)$ in $0.05 \mathrm{M}$ carbonate-bicarbonate buffer (pH 9.6) overnight at $4^{\circ} \mathrm{C}$, washed once with PBS contain- ing $0.05 \%$ Tween 20 (PBST), and blocked with $100 \mu \mathrm{l}$ of PBS diluted in $1 \% \mathrm{BSA}$ for $1 \mathrm{hr}$ at $37^{\circ} \mathrm{C}$. After washing, a 2 -fold serial dilution of bLF in PBS was added into the wells as a competitor and mixed with bLF-bio, bTF-bio or OTFbio at a concentration of $10 \mu \mathrm{g} / \mathrm{ml}$ in volumes of $50 \mu l$. After $1 \mathrm{hr}$ incubation at $37^{\circ} \mathrm{C}$, the plates were washed six times in PBST, and $50 \mu l$ streptavidin-HRP, diluted at $1: 10,000$ in PBS, was added into the wells and incubated for $1 \mathrm{hr}$ at $37^{\circ} \mathrm{C}$. The plates were washed six times with PBST, and $100 \mu \mathrm{l}$ of the substrate solution was added for color development. After $1 \mathrm{hr}$, the O. D. was read at $492 \mathrm{~nm}$ on a microplate reader.

Far Western blotting: Sodium dodecyl sulfate-polyacrylamide gel electrophoresis (SDS-PAGE) analysis was performed on a $10 \%$ polyacrylamide gel according to Laemmli's method [10]. The proteins were boiled for $5 \mathrm{~min}$ in sample buffer $(62.5 \mathrm{mM}$ Tris- $\mathrm{HCl} \mathrm{pH} 6.8,2 \%$ SDS, $5 \%$ $\beta$-mercaptoethanol, $10 \%$ glycerol, $0.02 \%$ bromophenol blue). After SDS-PAGE, the proteins were blotted onto a polyvinylidene fluoride (PVDF) membrane (Osmonics Inc., Westborough, MA). Electroblotting was performed for $1 \mathrm{hr}$ at room temperature in $25 \mathrm{mM}$ Tris, $192 \mathrm{mM}$ glycine, $20 \%$ ethanol buffer at $2 \mathrm{~mA} / \mathrm{cm}^{2}$. The blotted PVDF membrane was blocked with the blocking buffer (1\% BSA in PBS) for $1 \mathrm{hr}$ at room temperature. After blocking, the membrane was incubated with biotinylated proteins at a concentration of $1 \mu \mathrm{g} / \mathrm{m} l$ in the blocking buffer at $4^{\circ} \mathrm{C}$ overnight. The membrane was then washed five times in the washing buffer (0.05\% Tween 20 in PBS) and incubated at room temperature for 30 min with streptavidin-HRP diluted at 1:800 in the blocking buffer. Subsequently, the membrane was washed five times in the washing buffer and the protein bands were visualized on X-ray film using the ECL kit according to the manufacturer's protocol.

Competitive inhibition assay by the far Western blotting method: The blotted PVDF membrane, treated as described above, was blocked with the blocking buffer. After blocking, the membrane was incubated with bLF or bLF hydrolysate at the concentration $500 \mu \mathrm{g} / \mathrm{m} l$ in the blocking buffer, at room temperature for $30 \mathrm{~min}$. Then, biotinylated proteins were added to the competitor solution at a concentration of $1 \mu \mathrm{g} / \mathrm{m} l$ and the membrane was incubated at $4{ }^{\circ} \mathrm{C}$ overnight. The membrane was then washed five times in the washing buffer $(0.05 \%$ Tween 20 in PBS) and incubated at room temperature for $30 \mathrm{~min}$ with streptavidin-HRP diluted at 1:800 in the blocking buffer. Subsequently, the membrane was washed five times in the washing buffer and the protein bands were visualized on X-ray film using the ECL kit according to the manufacturer's protocol.

$N$-terminal amino acid sequence determination: $\mathrm{N}$-terminal amino acid sequences were determined using a gasphase sequencer 492A and chromatograph model 120A from Applied Biosystems (Foster City, CA).

\section{RESULTS}

Protein binding to T. brucei: We performed the fluores- 
A

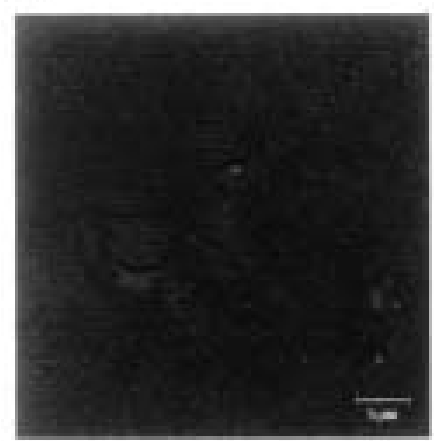

C

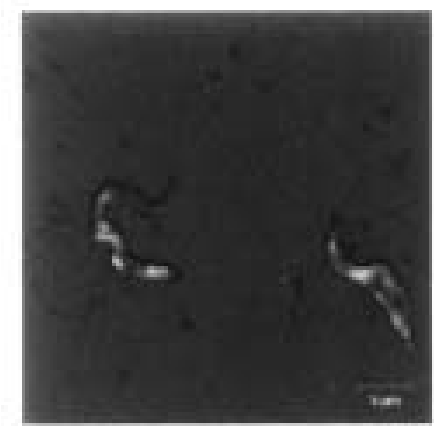

B

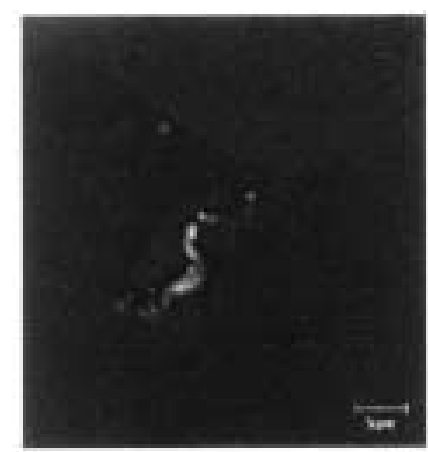

D

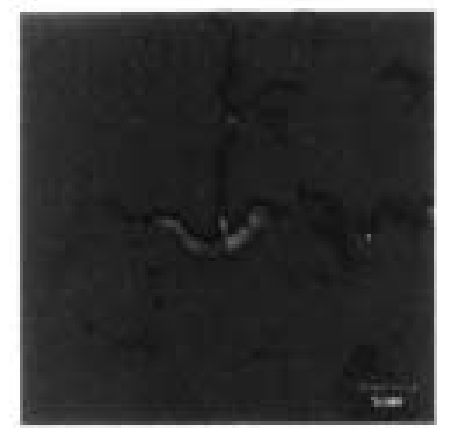

Fig. 1. Florescence staining of T. brucei using biotinylated proteins. After the parasites were reacted with controls (avidin-FITC only) (A), bLF-bio (B), bTFbio (C) and OTF-bio (D), the avidin-FITC reaction was observed by confocal laser microscopy.

cence test, SAB microplate analysis, and far Western blotting to confirm the binding of bLF, bTF, and OTF to $T$. brucei. In the fluorescence analysis, bLF-bio, bTF-bio, and OTF-bio reacted with $T$. brucei, but fluorescence intensity did not differ among the proteins (Fig. 1). Furthermore, bLF-bio, bTF-bio, and OTF-bio were analyzed by the SAB microplate method. O.D. values increased with increasing concentrations of bLF-bio, bTF-bio, and OTF-bio (Fig. 2). However, no significant differences in O.D. were observed between bLF-bio, bTF-bio, and OTF-bio at $10 \mu \mathrm{g} / \mathrm{m} l$ $(\mathrm{p}<0.05)$. Far Western blotting was performed to compare the binding pattern of holo-bLF-bio with that of apo-bLFbio on TNE buffer-, RIPA buffer-, and SDS lysis bufferextracted T. brucei proteins. Two bands (40 and $43 \mathrm{kDa}$ ) exhibited similar binding patterns in all three samples as demonstrated in Fig. 3. Therefore, we used apo-bLF-bio and soluble proteins extracted by SDS lysis buffer in the subsequent experiments. Figure 4 shows the results from far Western blotting of the $T$. brucei soluble proteins. Lane 1 exhibits the binding pattern of the soluble fraction using bLF-bio as the ligand. Two bands, 40 and $43 \mathrm{kDa}$, were observed. Lane 2 (hLF-bio), lane 3 (bTF-bio), and lane 4 (OTF-bio) also exhibit two bands (40 and $43 \mathrm{kDa}$ ) that were very similar to those observed for bLF-bio. The bLF bind-

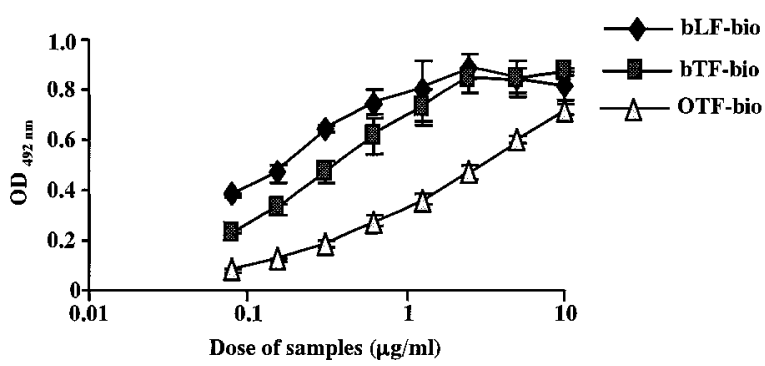

Fig. 2. bLF-bio, bTF-bio and OTF-bio binding assay. The wells were coated with whole $T$. brucei proteins, reacted with biotinylated proteins, and detected by streptavidin-HRP. Each value represents the mean \pm S.D. of triplicate samples.

ing proteins of $T$. brucei species (T. b. rhodesiense IL2343, T. b. gambiense, and T. evansi) are shown in Fig. 5. The molecular masses of the bLF binding proteins did not differ among $T$. brucei species.

Similarities in bLF-bio, bTF-bio, and OTF-bio binding: To study specific binding of bLF to $T$. brucei, we performed a competitive inhibition assay using 96-well plates. As shown in Fig. 6, the O.D. decreased when excess bLF was added as a competitor to the reaction mixtures. This result 
A

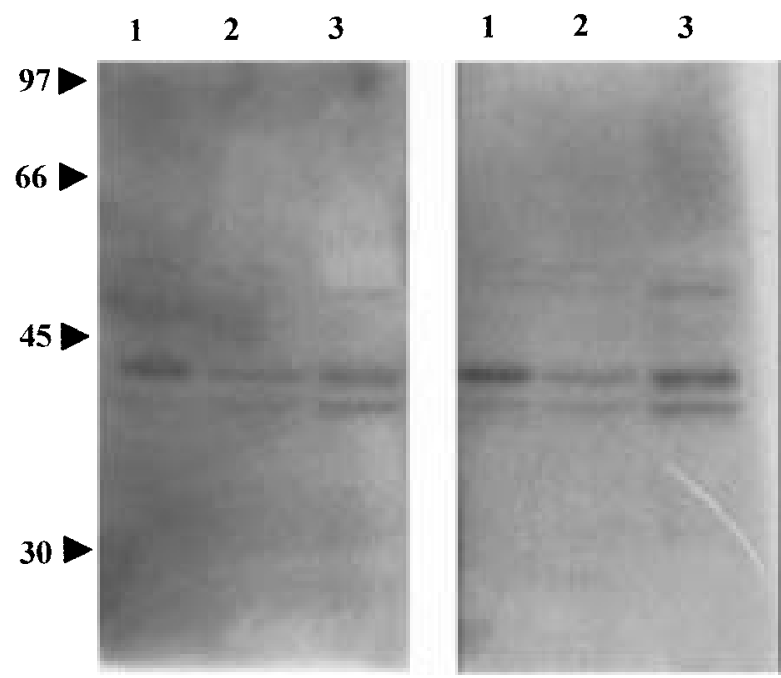

Fig. 3. The binding of bLF-bio (A) and holo-bLF-bio (B) to $T$. brucei proteins. The parasite proteins were extracted from $T$. brucei by TNE (lane 1), RIPA (lane 2) and SDS lysis (lane 3) buffer. The positions of molecular standards are indicated at the left, in $\mathrm{kDa}$.

indicated that bLF, bTF, and OTF recognize the same proteins. The competitive inhibition assay using far Western blotting demonstrated that the concentration of bLF increased 500 times over the first ligand. As shown in Fig. 7 , the bands in lane 2 were very faint when bLF was used as an competitor. However, a second competitive inhibition assay using bTF and OTF as the competitors did not reveal inhibition of bTF-bio and OTF-bio binding (data not shown). Likewise, the bLF pepsin hydrolysate did not completely inhibit the binding of bLF-bio to $T$. brucei (Fig. 8).

$\mathrm{N}$-terminal sequence analysis of the 40 and $43 \mathrm{kDa} b L F$ binding proteins of $T$. brucei: The $\mathrm{N}$-terminal amino acid residue of the $40 \mathrm{kDa}$ bLF binding protein was ${ }^{1}$ TIKVGINGFGRIGRM ${ }^{15}$. Comparison of the N-terminal amino acid residues derived from the $40 \mathrm{kDa}$ bLF binding protein with known sequences in the Swiss-Prot protein database indicated a high degree of homology $(100 \%)$ to the glycolytic enzyme glyceraldehyde-3-phosphate dehydrogenase (GAPDH) of T. brucei. However, we could not analyze the $43 \mathrm{kDa}$ protein band of $T$. brucei because it contained more than two proteins.

\section{DISCUSSION}

The TF family proteins, bLF, bTF, and OTF, exhibit approximately $60 \%$ identity in primary structure and are thus considered to share a close evolutionary relationship and have similar biological functions. On the other hand, $\mathrm{TF}$ release iron ions at $\mathrm{pH} 4.0$ but LF holds iron ions at $\mathrm{pH}>2.0$ and it is reported that the iron binding strength of $\mathrm{LF}$ is 260 -fold stronger than that of TF [1]. Therefore, it is

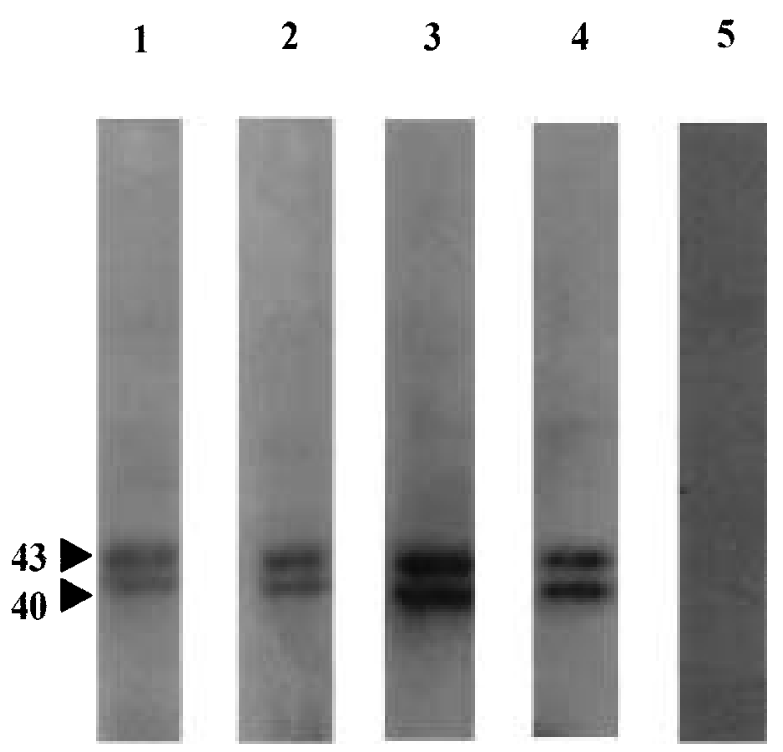

Fig. 4. The binding ability of bLF-bio (lane 1), hLF-bio (lane 2), bTF-bio (lane 3), OTF-bio (lane 4) and streptavidin-HRP only (lane 5) to $T$. brucei proteins. The parasite proteins were extracted from $T$. brucei by SDS lysis buffer. Molecular masses are given in $\mathrm{kDa}$.

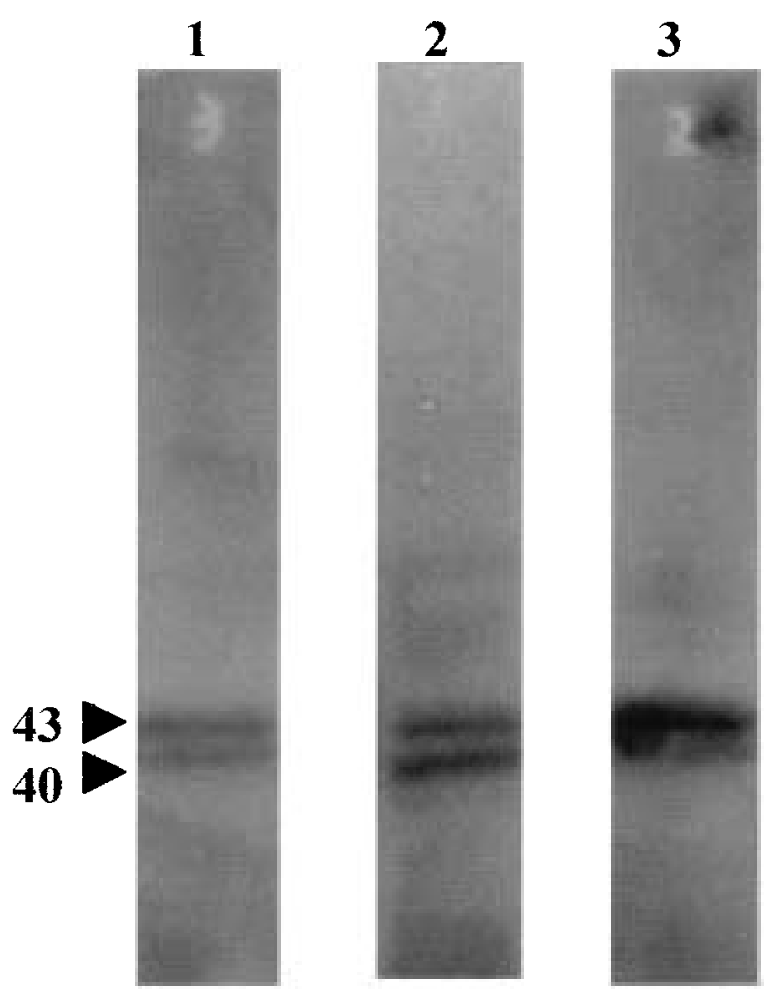

Fig. 5. The binding of bLF-bio to T. brucei species proteins (lane 1, T. b. rhodesiense IL2343; lane 2, T. b. gambiense; lane 3, T. evansi). The parasite proteins were extracted from $T$. brucei species by SDS lysis buffer. Molecular masses are given in $\mathrm{kDa}$. 


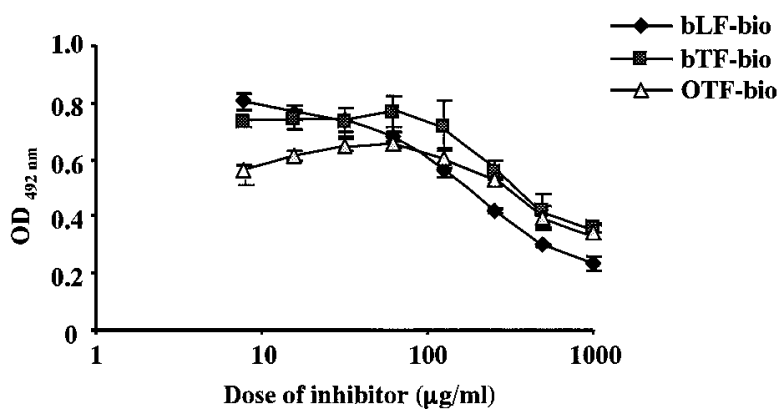

Fig. 6. A competitive inhibition assay for the binding of bLFbio, bTF-bio and OTF-bio to the T. brucei proteins using bLF as the competitor. The wells were coated with whole protein extracts from $T$. brucei. Then, the reaction using bLF as the competitor was performed. After reactions with bLF-bio, bTFbio, and OTF-bio, detection was performed with streptavidinHRP. Each value is the mean \pm S.D. of triplicate samples.
$\mathbf{A}$

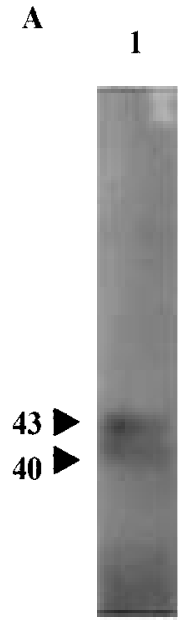

B

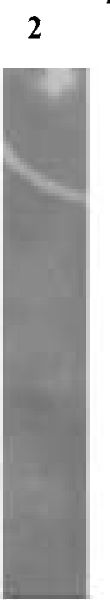

B

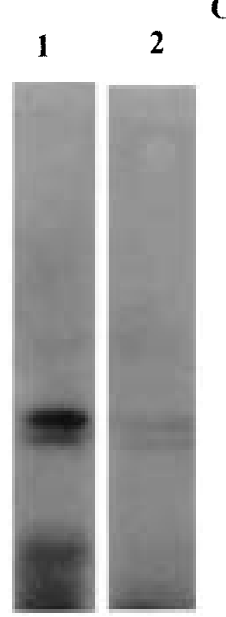

C

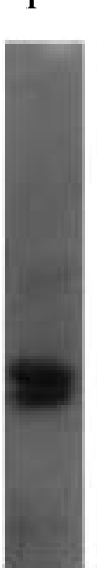

2

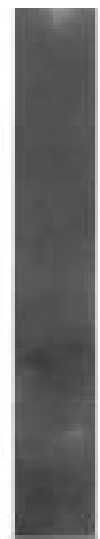

Fig. 7. A competitive inhibition assay of the binding ability of bLF-bio, bTF-bio, and OTF-bio to T. brucei proteins using bLF as the competitor. The parasite proteins were extracted from $T$. brucei by SDS lysis buffer. After reaction with BSA only (lane 1) or bLF (lane 2) at $500 \mu \mathrm{g} / \mathrm{ml}, 1 \mu \mathrm{g} / \mathrm{m} l$ bLF-bio (A), bTF-bio (B), and OTF-bio (C) were reacted with the membrane. Molecular masses are given in $\mathrm{kDa}$.

expected that the iron metabolism of $T$. brucei between LF and TF plays a role in different mechanism.

The fluorescence analysis using T. brucei fixed in methanol demonstrated the binding of bLF-bio, bTF-bio, and OTF-bio to BDFs (Fig. 1). These results suggested the presence of these binding proteins on the parasite. We used far Western blotting to confirm these observations. As shown in Fig. 3, only two protein bands were detected (40 and 43 $\mathrm{kDa}$ ) in the soluble fraction of T. brucei. The molecular masses of these proteins estimated from electrophoretic mobility were similar to those detected by hLF-bio, bTFbio, and OTF-bio (Fig. 4). However, it was not clear, whether these detected bands were identical. Therefore, we performed a competitive inhibition assay using two differ-

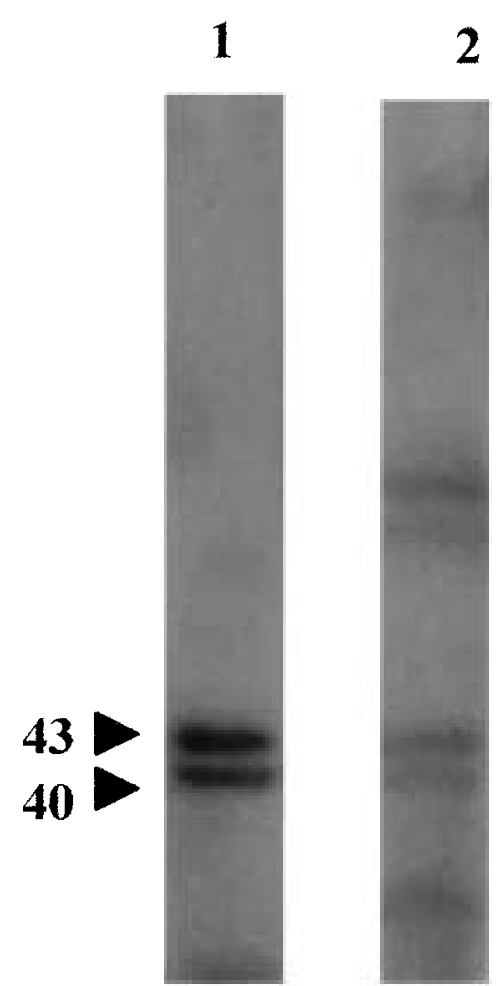

Fig. 8. A competitive inhibition assay of the binding ability of bLF-bio to T. brucei proteins using bLF hydrolysate as the competitor. Proteins were extracted from $T$. brucei by SDS lysis buffer. After reaction with BSA only (lane 1) or bLF hydrolysate (lane 2) at $500 \mu \mathrm{g} / \mathrm{m} l, 1 \mu \mathrm{g} / \mathrm{m} l$ bLF-bio was reacted with the membrane. Molecular masses are given in $\mathrm{kDa}$.

ent methods.

The first competitive inhibition assay was conducted according to the SAB microplate method using 96-well plates coated by the whole protein extract of T. brucei. As shown in Fig. 6, lower absorbance was observed when excess bLF as a competitor was added to the reaction mixtures. This suggested that bTF and OTF may bind to the same proteins as bLF. To test this, we added excess bTF and OTF to the reaction but no inhibition was observed (data not shown). Therefore, based on this assay, we concluded that bTF and OTF did not bind to the same protein as bLF. However, the possibility still existed that bLF-bio eliminated the bTF and OTF previously bound to the protein of interest. Because no band was observed in lane 2 of Fig. 7 , the results suggested that bLF-bio recognized the same protein bands as intact bLF. Consequently, a second competitive inhibition assay using bLF as an inhibitor of bTF-bio or OTF-bio binding to the soluble fraction of $T$. brucei was performed. Because inhibition was observed, we concluded that these three TF family proteins could recognize the 40 and $43 \mathrm{kDa}$ protein bands. The homologous primary struc- 
ture of these three TF family proteins suggests that the interaction with the binding proteins may occur through a common binding site. Furthermore, we demonstrated that bLF hydrolysate lost the peptide bond present at the parasite protein binding site (Fig. 8). These results indicated that the binding of bLF to $T$. brucei mean an importance to conserve three-dimensional structure or molecular size of bLF.

In order to identify the bLF binding proteins of T. brucei, we analyzed the N-terminal amino acid sequence of the 40 $\mathrm{kDa} \mathrm{bLF}$ binding protein. Based on the N-terminal amino acid sequence, the $40 \mathrm{kDa}$ protein was identified as GAPDH enzyme, which catalyzes the oxidation and phosphorylation of D-glyceraldehyde-3-phosphate to 1, 3-diphosphoglycerate [6]. T. brucei contains two isoenzymes for GAPDH; one enzyme resides in a microbody-like organelle, the glycosome, the other one is found in the cytosol [13]. The most of GAPDHs are generally considered soluble cytoplasmic proteins, a number of cell surface-associated GAPDHs in both prokaryotes and eukaryotes have been described. GAPDHs have, for example, been located in the cell membranes of human erythrocytes [9] and cell wall of Staphylococcus aureus [14]. It is known that $S$. aureus binds both TF [14] and LF [15]. In S. aureus, GAPDH has been shown to bind human TF. This suggests a potential means by which this bacterium is able to access iron in vivo [14]. Therefore, our results suggest that bLF may be involved in the metabolism of iron by T. brucei, although further experimental evidence is required to confirm this hypothesis.

TF uptake by $T$. brucei has been well characterized by many investigations. In this parasite, products of the ESAG6 and ESAG7 genes associate into a heterodimeric TF binding protein complex (TFBP) $[18,25]$. ESAG6 is a heavily glycosylated $50-60 \mathrm{kDa}$ molecule anchored to the membrane by a glycosyl-phosphatidylinositol anchor, whereas the $42 \mathrm{kDa}$ glycoprotein ESAG7 is unmodified at its $\mathrm{C}$-terminus. The TFBP complex can confer the ability to bind holo-TF, although neither ESAG6 nor ESAG7 alone can facilitate TF binding. Further characterization of the 43 $\mathrm{kDa}$ protein identified in the present study is required to determine its similarity to ESAG6 and ESAG7.

Binding of holo-TF to mammalian cell TF receptors is followed by internalization of receptor-ligand complexes and subsequent acidification in an endosomal compartment. Iron is released from $\mathrm{TF}$ under acidic conditions and is retained intracellularly, whereas apo-TF remains associated with its receptor and recycles back to the cell surface. Neutral $\mathrm{pH}$ causes dissociation of apo-TF from its receptor and promotes extracellular release. In contrast, the T. bruce $i$ TFBP-TF complex is internalized and transported to lysosomes, where TF is proteolytically degraded [25]. Thus, the trypanosomal and mammalian holo-TF uptake processes differ in the fate of internalized TF and in their dependence on $\mathrm{pH}$ for recycling. Pathogenic protozoa other than T. brucei utilize distinct pathways for iron uptake. Plasmodium falciparum can take up iron bound to TF and other chelators $[5,27,29]$. Likewise, there is evidence that TF binds to the surface of Trypanosoma cruzi and is subsequently internal- ized through an unknown mechanism [23]. The life cycles of $T$. vaginalis and $T$. foetus are limited to mucosal surfaces, and these protozoa bind LF, but not TF, in a receptor-mediated fashion $[17,26]$. Parasitic LF binding proteins potentially play a role in iron acquisition. However, we showed that iron saturation of bLF did not affect the binding pattern. Further studies are required to determine whether bLF and the binding protein promote or inhibit iron uptake in $T$. brucei.

ACKNOWLEDGEMENTS. This work was supported by grant from the Japan Society for the Promotion of Science (Grant-in-Aid for Encouragement of Young Scientists B) and the Akiyama Foundation. We are very grateful to $\mathrm{Mr}$. Yukichi Abe (Center for Instrumental Analysis, Hokkaido University) for technical assistance in N-terminal amino acid sequence determination.

\section{REFERENCES}

1. Aisen, P. and Leibman, A. 1972. Lactoferrin and transferrin: a comparative study. Biochim. Biophys. Acta 257: 314-323.

2. Blanton, K. J., Biswas, G. D., Tsai, J., Adams, J., Dayer, D. W., Davis, S. M., Koch, G. G., Sen, P. K. and Sparling, P. F. 1990. Genetic evidence that Neisseria gonorrhoeae produces specific receptors for transferrin and lactoferrin. J. Bacteriol. 172: 5225-5235.

3. Coppens, I., Opperdoes, F. R., Courtoy, P. J. and Baudhuin, P. 1987. Receptor-mediated endocytosis in the bloodstream form of Trypanosoma brucei. J. Protozool. 34: 465-473.

4. Grab, D. J., Lonsdale-Eccles, J. D., Oli, M. W. and Corbeil, L. B. 2001. Lactoferrin-binding proteins of Tritrichomonas foetus. J. Parasitol. 87: 1064-1070.

5. Haldar, K., Henderson, C. L. and Cross, G. A. 1986. Identification of the parasite transferrin-receptor of Plasmodium falciparum-infected erythrocytes and its acylation via 1,2-diacylsn-glycerol. Proc. Natl. Acad. Sci. U.S.A. 83: 8565-8569.

6. Harris, J. I. and Waters, M. 1976. The Enzymes (Boyer, P. D. ed.), Academic Press, New York.

7. Horwitz, D. A., Bakke, A. C., Abo, W. and Nishiya, K. 1984. Monocyte and NK cell cytotoxic activity in human adherent cell preparations: discriminating effects of interferon and lactoferrin. J. Immunol. 132: 2370-2374.

8. Isamida, T., Tanaka, T., Omata, Y., Yamauchi, K., Shimazaki, K. and Saito, A. 1998. Protective effects of lactoferricin against Toxoplasma gondii infection in mice. J. Vet. Med. Sci. 60: 241-244.

9. Kliman, H. J. and Steck, T. L. 1980. Association of glyceraldehyde-3-phosphate-dehydrogenase with the human red blood cell membrane. A kinetic analysis. J. Biol. Chem. 255: 63146321.

10. Laemmli, U. K. 1970. Cleavage of structural proteins during the assembly of the head of bacteriophage T4. Nature (Lond.) 227: 680-685.

11. Law, B. A. and Reiter, B. 1977. The isolation and bacteriostatic properties of lactoferrin from bovine milk whey. J. Dairy Res. 44: 595-599.

12. Lehker, M. W. and Alderete, J. F. 1992. Iron regulates growth of Trichomonas vaginalis and the expression of immunogenic trichomonad proteins. Mol. Microbiol. 6: 123-132.

13. Michels, P. A., Poliszczak, A., Osinga, K. A., Misset, O., Van 
Beeumen, J., Wierenga, R. K., Borst, P. and Opperdoes, F. R. 1986. Two tandemly linked identical genes code for the glycosomal glyceraldehyde-phosphate dehydrogenase in Trypanosoma brucei. EMBO J. 5: 1049-1056.

14. Modun, B. and Williams, P. 1999. The staphylococcal transferrin-binding protein is a cell wall glyceraldehyde-3-phosphate dehydrogenase. Infect. Immun. 67: 1086-1092.

15. Naidu, A. S., Andersson, M., Miedzobrodzki, J., Forsgren, A. and Watts, J. L. 1991. Bovine lactoferrin receptors in Staphylococcus aureus isolated from bovine mastitis. J. Dairy Sci. 74: $1218-1226$.

16. Nishiya, K. and Horwitz, D. A. 1982. Contrasting effects of lactoferrin on human lymphocyte and monocyte natural killer activity and antibody-dependent cell-mediated cytotoxicity. $J$. Immunol. 129: 2519-2523.

17. Peterson, K. M. and Alderete, J. F. 1984. Iron uptake and increased intracellular enzyme activity follow host lactoferrin binding by Trichomonas vaginalis receptors. J. Exp. Med. 160: 398-410.

18. Salmon, D., Geuskens, M., Hanocq, F., Hanocq-Quertier, J., Nolan, D., Ruben, L. and Pays, E. 1994. A novel heterodimeric transferrin receptor encoded by a pair of VSG expression siteassociated genes in T. brucei. Cell 78: 75-86.

19. Schell, D., Borowy, N. K. and Overath, P. 1991. Transferrin is a growth factor for the bloodstream form of Trypanosoma brucei. Parasitol. Res. 77: 558-560.

20. Schryvers, A. B. 1989. Identification of the transferrin- and lactoferrin- binding proteins in Haemophilus influenzae. J. Med. Microbiol. 29: 121-130.
21. Schryvers, A. B. and Morris, L. J. 1988. Identification and characterization of the human lactoferrin-binding protein from Neisseria meningitidis. Infect. Immun. 56: 1144-1149.

22. Shimazaki, K. 2000. Lactoferrin: A marvelous protein in milk? Anim. Sci. J. 71: 329-347.

23. Soares, M. J., Souto-Padron, T. and De Souza, W. 1992. Identification of a large pre-lysosomal compartment in the pathogenic protozoan Trypanosoma cruzi. J. Cell Sci. 102: 157-167.

24. Steverding, D. 1998. Bloodstream forms of Trypanosoma brucei require only small amounts of iron for growth. Parasitol. Res. 84: 59-62.

25. Steverding, D., Stierhof, Y. D., Fuchs, H., Tauber, R. and Overath, P. 1995. Transferrin-binding protein complex is the receptor for transferrin uptake in Trypanosoma brucei. J. Cell Biol. 131: 1173-1182.

26. Tachezy, J., Suchan, P., Schrevel, J. and Kulda, J. 1998. The host-protein-independent iron uptake by Tritrichomonas foetus. Exp. Parasitol. 90: 155-163.

27. Tsafack, A., Golenser, J., Libman, J., Shanzer, A. and Cabantchik, Z. I. 1995. Mode of action of iron (III) chelators as antimalarials. III. Overadditive effects in the combined action of hydroxamate-based agents on in vitro growth of Plasmodium falciparum. Mol. Pharmacol. 47: 403-409.

28. Voyiatzaki, C. S. and Soteriadou, K. P. 1992. Identification and isolation of the Leishmania transferrin receptor. J. Biol. Chem. 267: 9112-9117.

29. Wilson, M. E. and Britigan, B. E. 1998. Iron acquisition by parasitic protozoa. Parasitol. Today 14: 348-353. 